\title{
Low temperature silicon nitride waveguides for multilayer platforms
}

\author{
T. Domínguez Bucio, A. Tarazona, A. Z. Khokhar, G. Z. Mashanovich, F. Y. Gardes. \\ Optoelectronics Research Centre, University of Southampton, Highfield Campus, SO171BJ, Southampton,UK
}

\begin{abstract}
Several 3-D multilayer silicon photonics platforms have been proposed to provide densely integrated structures for complex integrated circuits. Amongst these platforms, great interest has been given to the inclusion of silicon nitride layers to achieve low propagation losses due to their capacity of providing tight optical confinement with low scattering losses in a wide spectral range. However, none of the proposed platforms have demonstrated the integration of active devices. The problem is that typically low loss silicon nitride layers have been fabricated with LPCVD which involves high processing temperatures $\left(>1000^{\circ} \mathrm{C}\right)$ that affect metallisation and doping processes that are sensitive to temperatures above $400{ }^{\circ} \mathrm{C}$. As a result, we have investigated ammonia-free PECVD and HWCVD processes to obtain high quality silicon nitride films with reduced hydrogen content at low temperatures. Several deposition recipes were defined through a design of experiments methodology in which different combinations of deposition parameters were tested to optimise the quality and the losses of the deposited layers. The physical, chemical and optical properties of the deposited materials were characterised using different techniques including ellipsometry, SEM, FTIR, AFM and the waveguide loss cut-back method. Silicon nitride layers with hydrogen content between 10-20\%, losses below $10 \mathrm{~dB} / \mathrm{cm}$ and high material quality were obtained with the ammonia-free recipe. Similarly, it was demonstrated that HWCVD has the potential to fabricate waveguides with low losses due to its capacity of yielding hydrogen contents $<10 \%$ and roughness $<1.5 \mathrm{~nm}$.
\end{abstract}

Keywords: Multilayer platforms, silicon nitride, low temperature, PECVD, HWCVD, waveguides, hydrogen content, propagation losses.

\section{INTRODUCTION}

Multilayer photonic platforms have the potential to provide the means to fabricate complex and densely integrated photonic circuits that can be used for a wide range of applications. In diverse works, efforts have been directed to develop multilayer platforms with low propagation and coupling losses based on silicon-on-insulator substrates $^{1}$ and the integration of different materials such as silicon oxide, ${ }^{2}$ silicon nitride (SiN) ${ }^{3,4}$ polysilicon ${ }^{5}$ and amorphous silicon ${ }^{6}$ on standard silicon substrates. Amongst the proposed platforms, great interest has been given to SiN as an alternative to silicon for sensing, ${ }^{7}$ nonlinear applications, ${ }^{8}$ and passive devices working in the visible, near-infrared and mid-infrared wavelength regimes. ${ }^{9,10}$

In particular, $\mathrm{SiN}$ is an attractive material for passive waveguide routing in multilayer platforms for optical interconnects because of the low propagation losses that can be achieved with this material due to its capacity of providing tight optical confinement with low scattering losses ${ }^{11}$ and that have already been demonstrated in previous works with SiN waveguides fabricated through low-pressure chemical vapour deposition (LPCVD). ${ }^{12}$ However, the deposition of LPCVD SiN layers involves high processing temperatures $\left(>1000^{\circ} \mathrm{C}\right)$ that limit the fabrication of active devices that are of critical importance to obtain the complete functionality of multilayer photonic circuit platforms as they require metallisation and doping processes sensitive to temperatures $>400^{\circ} \mathrm{C}$. Alternatively, SiN layers have been deposited through plasma-enhanced chemical vapour deposition (PECVD) at lower temperatures $\left(400^{\circ} \mathrm{C}\right)$. The issue with PECVD SiN layers is that they are prone to high propagation losses in the Telecom wavelengths because they suffer from substantial hydrogen $(\mathrm{H})$ incorporation in the form of $\mathrm{N}-\mathrm{H}$ and $\mathrm{Si}-\mathrm{H}$ bonds that act as absorption centres in this spectral region because of their lower processing

Silicon Photonics and Photonic Integrated Circuits V, edited by Laurent Vivien, Lorenzo Pavesi, Stefano Pelli, Proc. of SPIE Vol. 9891, 98911T · C 2016 SPIE · CCC code: 0277-786X/16/\$18 · doi: 10.1117/12.2227590 
temperatures. ${ }^{13}$ Hence, to achieve the low propagation losses desirable for multilayer platforms, PECVD layers must be subjected to high temperature annealing processes $\left(>400^{\circ} \mathrm{C}\right)$ to reduce considerably the $\mathrm{H}$ impurities present in the films. ${ }^{14}$ As a result, none of the previously described approaches is viable for incorporating $\mathrm{SiN}$ films in multilayer platforms.

In this work, we have investigated two alternative deposition processes to fabricate SiN films for multilayer platforms with potentially low propagation losses at temperatures below $<400^{\circ} \mathrm{C}$. The first process is a modified PECVD recipe that uses $\mathrm{N} 2$ as a precursor gas instead of $\mathrm{NH} 3$ to reduce the $\mathrm{H}$ incorporation in the deposited films without the need of high temperature annealing as demonstrated by Karouta et al. ${ }^{15}$ The second alternative is the deposition process known as hot-wire chemical vapour deposition which has been used to obtain SiN films with low hydrogen content for solar cell applications. ${ }^{16}$ Contrary to other works in literature, we have studied the properties of the deposited films that allow evaluating how suitable each technique is for multilayer photonic platforms rather than only focusing on the level of $\mathrm{H}$ incorporation achieved. These properties are mainly the material quality, thickness uniformity, roughness, $\mathrm{H}$ content, and most importantly the propagation losses of each deposited film.

\section{EXPERIMENTAL WORK}

As a first step, several recipes in which the deposition parameters were varied following a design of experiments methodology were tested in order to reduce the $\mathrm{H}$ content of the films deposited with each of the studied techniques. For this purpose, SiN films were grown on 2x2cm p-type $\mathrm{Si}$ samples with a maximum processing temperature of $350^{\circ} \mathrm{C}$. The NH3-free films were deposited using the Oxford Plasma Technology 100 PECVD system with pressure of $650 \mathrm{~m}$ Torr and fixed $\mathrm{N} 2$ flow of $980 \mathrm{sccm}$. In this case, the power of the PECVD radio frequency generator $(\mathrm{RF})$ and the $\mathrm{SiH} 4$ flow were varied in different experiments. On the other hand, the HWCVD films were deposited using the Echerkon Nitor 301 system with a pressure of $25 \mathrm{mTorr}$ and a fixed $\mathrm{SiH} 4$ flow of $6 \mathrm{sccm}$. For this technique, the experiments had as controllable parameters the temperature of the filaments (TF) and the NH3 flow.

The deposition rate, thickness and material quality of the different SiN samples were estimated from crosssectional scanning electron microscopy (SEM) images of the deposited films. Their surface roughness was characterised quantitatively by calculating the root-mean-square roughness (RMS) over an area of $2 \times 2 \mu \mathrm{m}$ using atomic force microscopy (AFM) on tapping mode with a Si cantilever. The refractive index at $632.8 \mathrm{~nm}$ and thickness uniformity of the samples were characterized using a M2000DI ellipsometer. Then, the N/Si ratio of the different films was estimated from the measured refractive index using the procedure described by Mackel et. al. ${ }^{17}$ Finally, the hydrogen absorption peaks of N-H $(\sim 3350 \mathrm{~cm}-1)$ and Si-H $(\sim 2200 \mathrm{~cm}-1)$ bonds along with the total hydrogen content of the SiN films were examined by Fourier transform infrared spectroscopy (FTIR) using a Varian 600 FTIR spectrometer. In this case, the hydrogen concentration of the deposited films was quantitatively estimated from the measured FTIR spectra using the method described by Yin and Smith. ${ }^{18}$

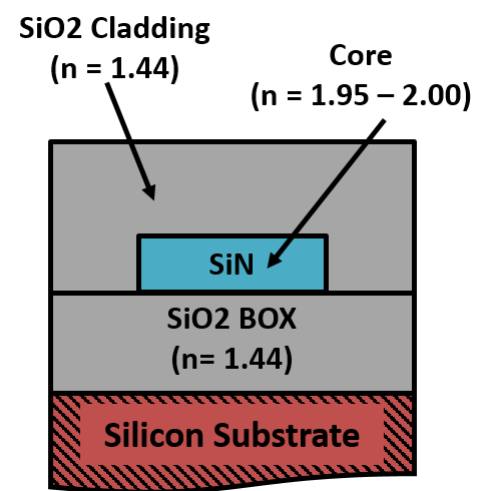

Figure 1. Schematic of the geometry for the SiN waveguides used to measure the propagation losses of the deposited layers. 

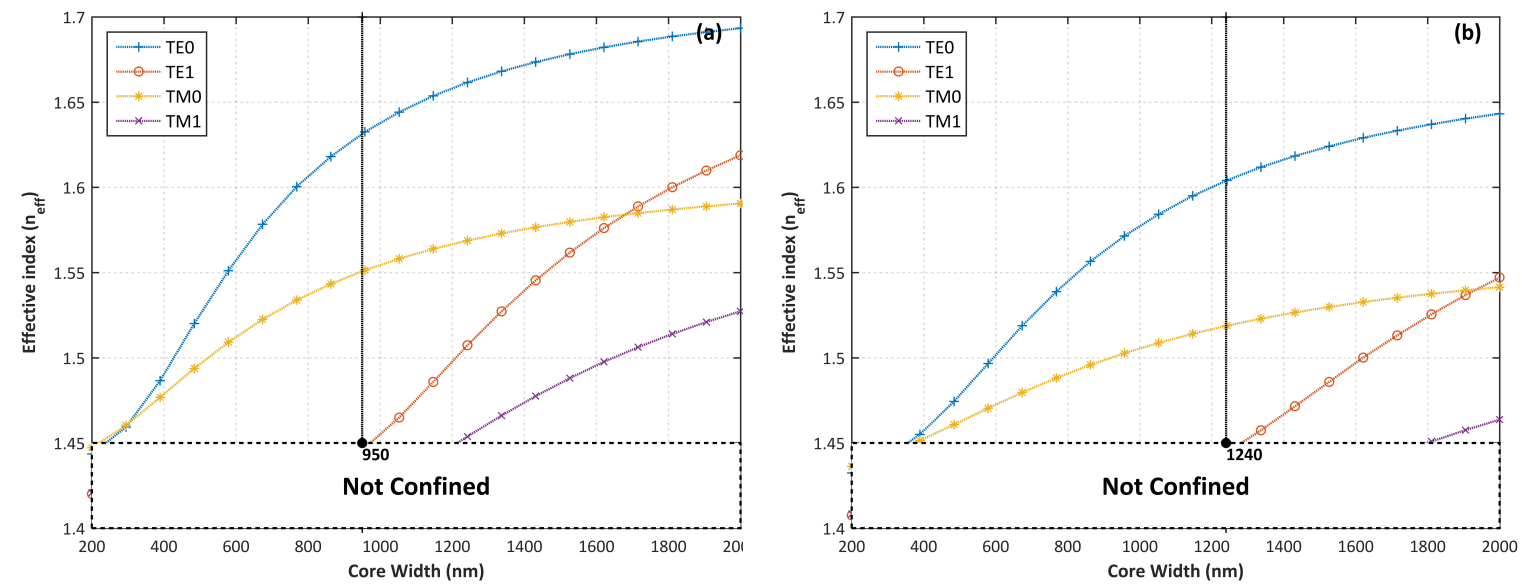

Figure 2. Dispersion curves for 300nm thick SiN waveguides at (a) 1310nm and (b) 1550nm. The single mode operation region is indicated by the cutoff frequency for TE polarisation.

Some of the evaluated SiN films were selected to fabricate waveguides with a geometry similar to the one presented in Fig. 1 in order to quantify the propagation losses of the materials grown with both deposition techniques at $1310 \mathrm{~nm}$ and $1550 \mathrm{~nm}$. In order to assure single mode operation, the dispersion curves for $300 \mathrm{~nm}$ $\mathrm{SiN}$ waveguides with a $\mathrm{SiO} 2$ cladding were calculated using Lumerical Mode Solutions at both wavelengths assuming a refractive index of 2.00 for $\mathrm{SiN}$. As shown in Fig. 2, the waveguides have a single mode behaviour for TE polarisation with widths below $950 \mathrm{~nm}$ for $1310 \mathrm{~nm}$ and below $1240 \mathrm{~nm}$ for $1550 \mathrm{~nm}$. Hence, the widths selected for the fabricated waveguides were of 900 and $1200 \mathrm{~nm}$ for operation at 1310 and $1550 \mathrm{~nm}$ respectively. Similarly, fully-etched grating couplers were designed to couple TE polarised light at 1310 and $1550 \mathrm{~nm}$ using the defined waveguide thickness of $300 \mathrm{~nm}$ and the selected widths for each wavelength. In this case, the bottom oxide for the grating couplers was selected to be $3.26 \mu \mathrm{m}$ in order to avoid leakage into the substrate and to optimize the grating coupler efficiency with a top oxide cladding of 530nm. A maximum theoretical coupling efficiency of $58 \%$ was obtained at $1310 \mathrm{~nm}$ with a grating period of $1 \mu \mathrm{m}$ and filling factor of $50 \%$, whereas a maximum theoretical coupling efficiency of $40 \%$ was obtained at $1550 \mathrm{~nm}$ with a period of $1.2 \mu \mathrm{m}$ and $50 \%$ filling factor.

To measure the propagation losses two sets of 20 straight SiN waveguides with the selected widths and lengths up to $1.9 \mathrm{~cm}$ were fabricated on $6 "$ p-type Si wafers. As a first layer, a $3.26 \mu \mathrm{m}$ thick PECVD $\mathrm{SiO} 2$ layer was deposited at $350^{\circ} \mathrm{C}$ on the bare Si wafers using an Oxford Plasma Technology 100 PECVD reactor to act as bottom cladding for the waveguides and grating couplers. On top of the bottom oxide, 300nm thick SiN layers were deposited using the selected recipes for NH3-free PECVD and HWCVD using a maximum processing temperature of $350^{\circ} \mathrm{C}$ to ensure CMOS back-end compatibility. The refractive index and thickness of the deposited SiN layers were measured with ellipsometry as it was done before with the samples in order to make sure that their thickness was close to the desired $300 \mathrm{~nm}$. Afterwards, the devices were patterned using electron beam lithography with a 450nm ZEP resist that was spun and baked on top of the SiN layer. Once the pattern was developed, it was transferred to the SiN layer using an inductive coupled plasma-reactive ion-etch process performed with an Oxford Plasmalab 100 ICP etcher using a fluorine-based chemistry with SF6 and CHF3 that was optimized to obtain nearly vertical side walls. Finally, the waveguides were covered with a 530nm PECVD $\mathrm{SiO} 2$ cladding deposited using the same process used for the bottom cladding. A top view of an etched grating coupler with a period of $1 \mu \mathrm{m}$ can be seen in Fig. 3 .

Finally, the propagation losses were obtained using the cutback method by measuring individually the spectral response of each waveguide with different length. The experimental set-up that was used for this purpose included two tunable laser sources with continuous sweep mode centred at 1310 (Agilent 8164B Lightwave Measurement System) and 1550nm (Agilent 8163B Lightwave Multimeter). The output of the laser sources was connected to a polarisation maintaining fibre (PM) that was coupled to a normal fibre with a polarisation controller that allowed adjusting the polarisation of the input light to maximise the output. The fibre was then fixed to a 


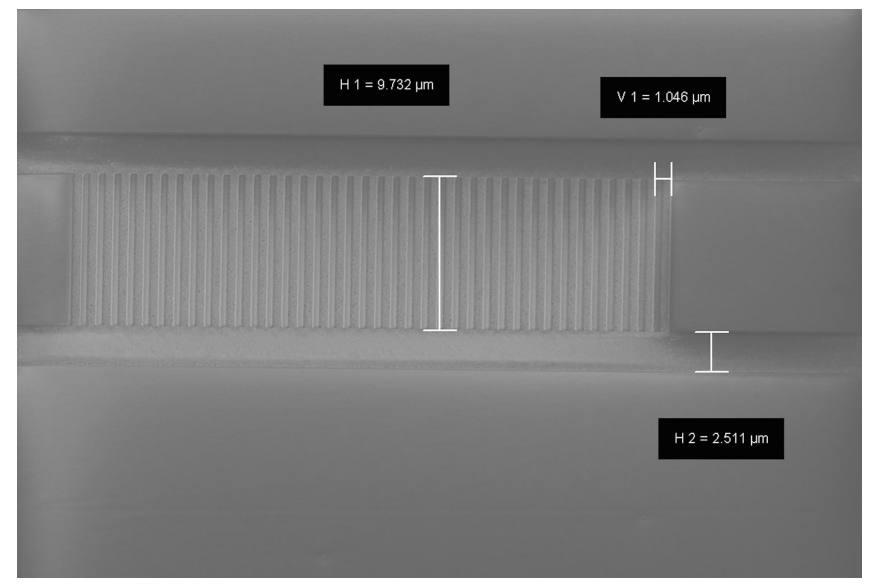

Figure 3. Top view SEM of a grating coupler fabricated with a $1 \mu \mathrm{m}$ period..

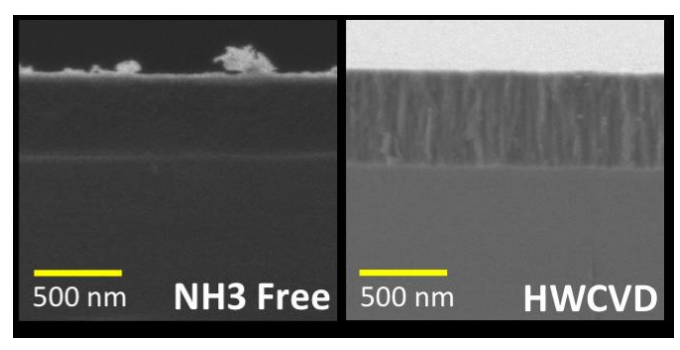

Figure 4. SEM images showing the material structure of NH3-free and HWCVD SiN layers.

support placed on a translation stage that allowed adjusting both the angle of incidence and the position of the fibre on top of the device chips, so that the light coupled through the gratings could be maximised. The light at the output of the measured waveguides was then coupled back into another fibre connected to a power meter. A camera was placed on top of the chips to visualize the devices and optimize the alignment between the fibres and the grating couplers.

\section{RESULTS}

Figure 4 shows SEM images with the cross-section of SiN layers deposited with NH3-free PECVD and HWCVD. It was observed that the films deposited with both techniques presented the classic micro-columnar structure expected of amorphous layers. However, the structure of the HWCVD layers showed a higher density of microcolumns. This means that the material quality of the deposited HWCVD layers is lower than that of NH3-free PECVD layers and, so, these films are prone to higher propagation losses due to the increased amount of scattering centres within their structure. This result can be attributed to the lower energy that the reactive species have when arriving to the substrate due to the nature of the HWCVD deposition process.

The experiments also demonstrated that in a wafer scale NH3-free PECVD layers have a non-uniformity $>10 \%$ that is dependant on the deposition conditions and that is two times higher than the non-uniformity of $5 \%$ obtained with HWCVD layers regardless of the deposition recipe used. Also, as it can be observed in Fig. 5, the thickness measurements taken with ellipsometry showed that the grown HWCVD layers have a non-uniformity $<1 \%$ in the central area of the wafer. These results suggest HWCVD layers have a uniformity that is independent of the deposition conditions which is ideal to achieve uniformity over large areas with the advantage of having the possibility of changing the deposition parameters without affecting the overall uniformity of the deposited layers, whereas the uniformity of NH3-free PECVD layers is strongly affected by the deposition conditions. 

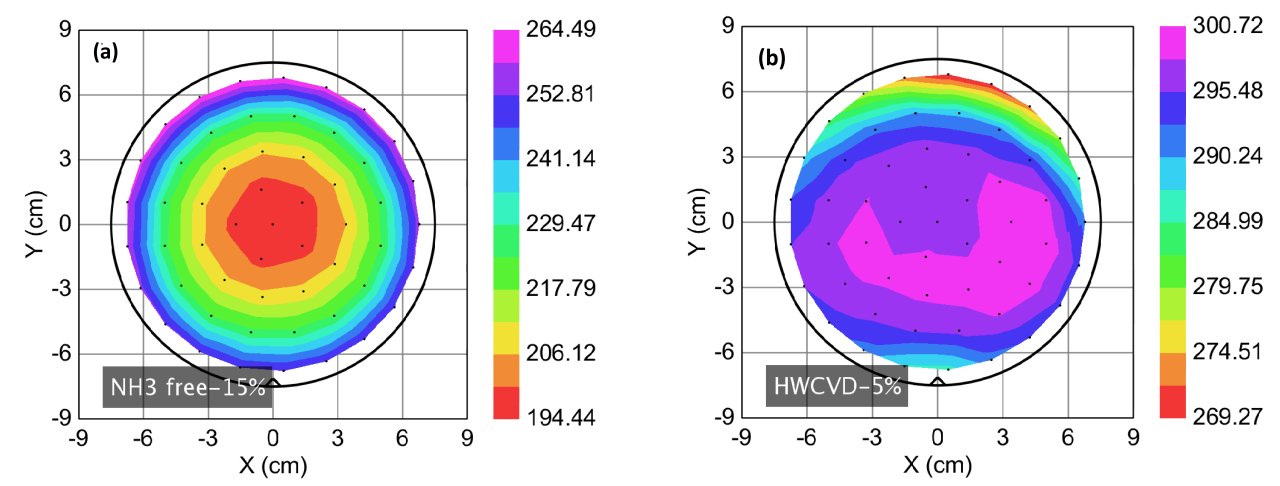

Figure 5. Thickness uniformity measured by ellipsometry for (a) NH3-free PECVD and (b) HWCVD layers.

(a)

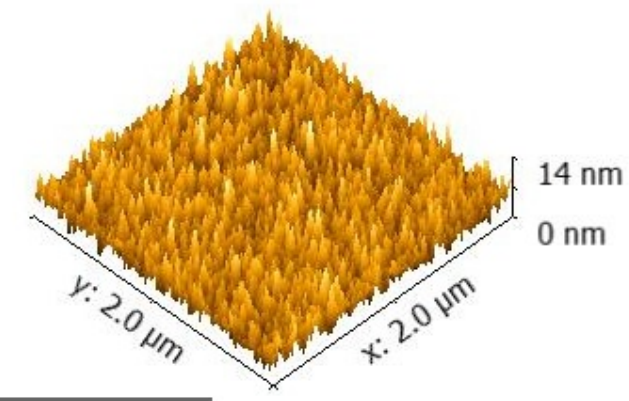

Rms $=2.49$ (b)

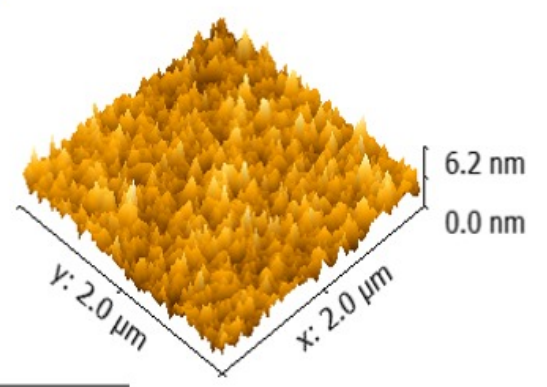

Rms $=0.61$

Figure 6. 3D AFM micrographs of SiN films deposited with (a) NH3-free PECVD and (b) HWCVD.

The AFM measurements in Fig. 6 showed that the lowest RMS roughness of $0.61 \mathrm{~nm}$ was obtained with HWCVD films, whereas NH3-free films had a minimum RMS roughness of $2.49 \mathrm{~nm}$. This result can be explained by the lack of ion bombardment in the HWCVD process that increases the roughness of NH3-free PECVD layers. Also, the results suggest that the roughness of the fabricated waveguides came mostly from the $\mathrm{SiO} 2$ layer underneath the SiN, which had a RMS value of about $4 \mathrm{~nm}$.

The experiments conducted in this work demonstrated that the N/Si ratio of the deposited films can be tuned between 0.6 and 1.70 by changing the deposition conditions with either of the studied techniques. More important, the results showed that the total $\mathrm{H}$ concentration and the propagation losses of the deposited films have an intrinsic relation with their $\mathrm{N} / \mathrm{Si}$ ratio. In this case, both of them decrease with increasing $\mathrm{N} / \mathrm{Si}$ ratio until they reach a minimum value near the stoichiometric $\mathrm{N} / \mathrm{Si}$ value (1.33) and then increase back again as the $\mathrm{N} / \mathrm{Si}$ ratio keeps increasing. Under this context, films with lower $\mathrm{H}$ concentration and with a $\mathrm{N} / \mathrm{Si}$ ratio close to stoichiometry are expected to have lower propagation losses at both wavelengths. As it can be observed in the FTIR spectra in figure 7, the minimum $\mathrm{H}$ concentration obtained with both techniques is lower that the typical $\mathrm{H}$ concentration $>20$ at.\% observed on standard PECVD layers. In fact, the lowest $\mathrm{H}$ concentration of 9.7 at.\% was obtained with the HWCVD deposition process followed closely by NH3-free layers with a minimum $\mathrm{H}$ concentration of 18 at.\%. From this result, it would be expected that HWCVD layers had lower propagation losses as they have lower H\%. However, as it can be seen in Fig. 8, the lowest losses of $5.1 \mathrm{~dB} / \mathrm{cm} @ 1550 \mathrm{~nm}$ and $2.0 \mathrm{~dB} / \mathrm{cm} @ 1310 \mathrm{~nm}$ were obtained with NH3-free PECVD, while the losses obtained with HWCVD were of $12.3 \mathrm{~dB} / \mathrm{cm} @ 1550 \mathrm{~nm}$ and $7.1 \mathrm{~dB} / \mathrm{cm} @ 1310 \mathrm{~nm}$. In both cases, the films with the lowest propagation losses were films with a N/Si ratio close to stoichiometry. The higher losses in the HWCVD layers which had a lower $\mathrm{H}$ concentration than NH3-free PECVD layers can be explained by the lower material quality of the HWCVD films 


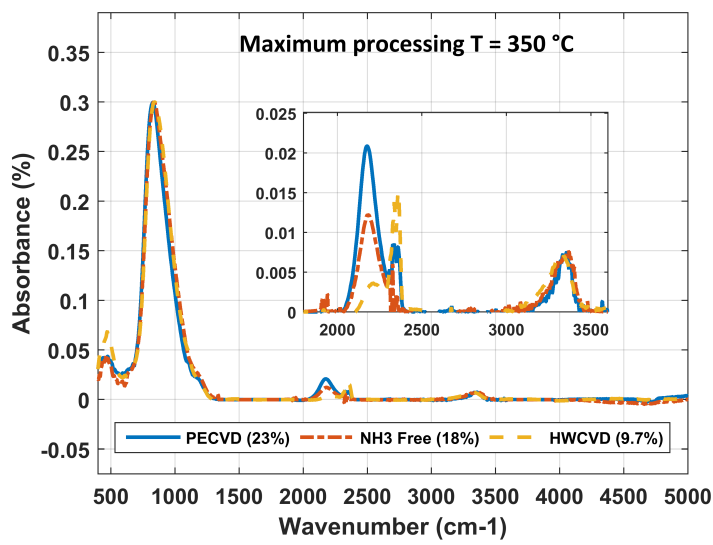

Figure 7. FTIR spectra of PECVD, NH-free PECVD and HWCVD SiN layers showing the Si-N peak at $\sim 860 \mathrm{~cm}-1$, the $\mathrm{Si}-\mathrm{H}$ peak at $\sim 2200 \mathrm{~cm}-1$, and the N-H peak at $\sim 3300 \mathrm{~cm}-1$. The inset zooms in the Si-H and N-H peaks.
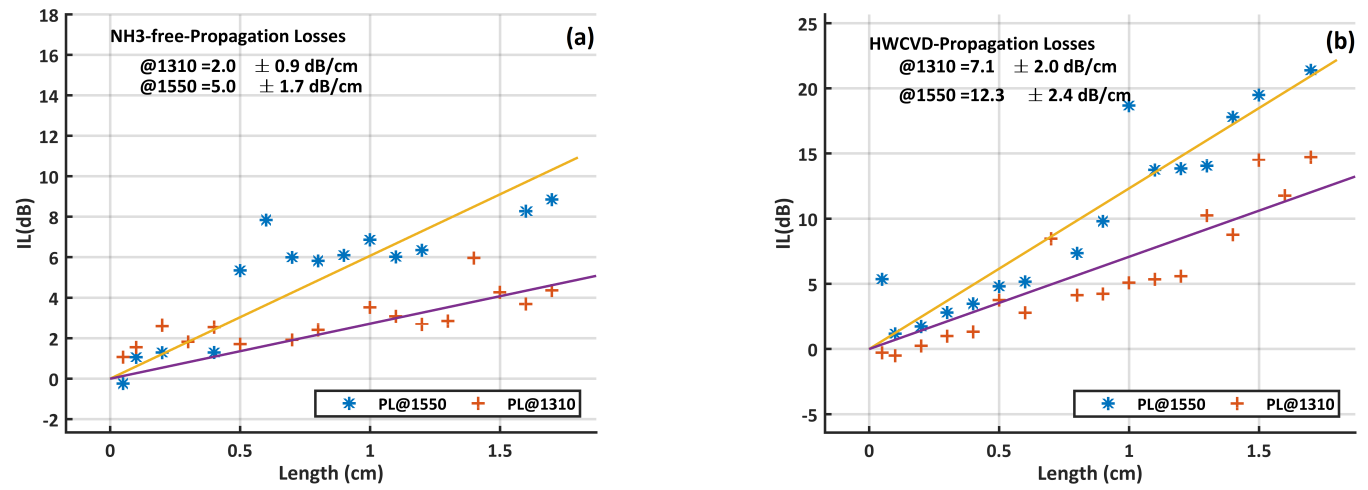

Figure 8. Insertion loss as a function of waveguide length at 1310 and 1550nm for the selected (a) NH3-free PECVD and (b) HWCVD films.

which have more interfaces that can scatter light producing higher propagation losses.

\section{CONCLUSIONS}

In this work, we have demonstrated that both NH3-free and HWCVD deposition techniques are viable for fabricating SiN films for multilayer platforms because they allow tuning the stoichiometry of the films to fulfil the requirements of different applications while having a low processing temperature $\left(<400^{\circ} \mathrm{C}\right)$. Single mode waveguides with losses below $2 \mathrm{~dB} / \mathrm{cm} @ 1310 \mathrm{~nm}$ and around $5 \mathrm{~dB} / \mathrm{cm} @ 1550 \mathrm{~nm}$ were obtained with NH3-free PECVD SiN layers, while losses of $12.3 \mathrm{~dB} / \mathrm{cm} @ 1550 \mathrm{~nm}$ and $7.1 \mathrm{~dB} / \mathrm{cm} @ 1310 \mathrm{~nm}$ were measured with waveguides fabricated on HWCVD SiN layers. NH3-free layers exhibited a higher material quality, but higher non-uniformity and surface roughness possibly due to the ion bombardment that occurs during the deposition process. On the other hand, despite their higher propagation losses, HWCVD SiN layers are still a promising approach for multilayer platforms because of their higher uniformity that is independent of the deposition conditions and their lower surface roughness. Hence, studying further how the deposition parameters affect the results obtained with the HWCVD layers can help improve the material quality of the films to fabricate waveguides with propagation losses potentially lower than those observed with the NH3-free PECVD layers. 


\section{REFERENCES}

1. Koonath, P. and Jalali, B., "Multilayer 3-D Photonics in Silicon," OFC/NFOEC , 1-3 (2007).

2. Tsuchizawa, T., Yamada, K., Watanabe, T., Park, S., Nishi, H., Kou, R., Shinojima, H., and Itabashi, S., "Monolithic Integration of Silicon-, Germanium-, and Silica-Based Optical Devices for Telecommunications Applications," IEEE Journal of Selected Topics in Quantum Electronics 17(3), 516-525 (2011).

3. Huang, Y., Song, J., Luo, X., Liow, T.-Y., and Lo, G.-Q., "CMOS compatible monolithic multi-layer Si3N4on-SOI platform for low-loss high performance silicon photonics dense integration," Opt. Express 22(18), 21859-21865 (2014).

4. Sacher, W. D., Huang, Y., Lo, G., and Poon, J. K. S., "Multilayer Silicon Nitride-on-Silicon Integrated Photonic Platforms and Devices," IEEE/OSA Journal of Lightwave Technology 33(4), 901-910 (2015).

5. Kwong, D., Hosseini, A., Covey, J., Zhang, Y., Xu, X., and Chen, R. T., "Low loss polycrystalline silicon waveguides and devices for multilayer on-chip optical interconnects," Proc. SPIE 8630, 863012-863012-8 (2013).

6. Kang, J., Atsumi, Y., Oda, M., Amemiya, T., Nishiyama, N., and Arai, S., "Low-loss amorphous silicon multilayer waveguides vertically stacked on silicon-on-insulator substrate," Japanese Journal of Applied Physics 50(12R), 120208 (2011).

7. Neutens, P., Subramanian, A., Ul Hasan, M., Chen, C., Jansen, R., Claes, T., Rottenberg, X., Du Bois, B., Leyssens, K., Helin, P., Severi, S., Dhakal, A., Peyskens, F., Lagae, L., Deshpande, P., Baets, R., and Van Dorpe, P., "Characterization of PECVD silicon nitride photonic components at 532 and $900 \mathrm{~nm}$ wavelength," Proc. SPIE 9133, 91331F-91331F-6 (2014).

8. Moss, D. J., Morandotti, R., Gaeta, A. L., and Lipson, M., "New CMOS-compatible platforms based on silicon nitride and Hydex for nonlinear optics," Nature Photonics 7(8), 597-607 (2013).

9. Soref, R., "Mid-infrared photonics in silicon and germanium," Nature Photonics 4, 495-497 (2010).

10. Subramanian, A. Z., P.Neutens, Dhakal, A., Jansen, R., T.Claes, Rottenber, X., Peyskens, F., Selvaraja, S., Helin, P., Bois, B. D., K.Leyssens, Severi, S., Deshpande, P., Baets, R., and Dorpe, P. V., "Low-loss singlemode PECVD silicon nitride photonic wire waveguides for 532-900nm wavelength window fabricated within a CMOS pilot line," IEEE Photonics Journal 5(6), 2202809-2202809 (2013).

11. Daldosso, N., Melchiorri, M., Riboli, F., Girardini, M., Pucker, G., Crivellari, M., Belutti, P., Lui, A., and Pavesi, L., "Comparison Among Various Si3N4 Waveguide Geometries Grown within a CMOS Fabrication Pilot Line," IEEE/OSA Journal of Lightwave Technology 22(7), 1734-1740 (2004).

12. Melchiorri, M., Daldosso, N., Sbrana, F., Pavesi, L., Pucker, G., Kompocholis, C., Belluti, P., and Lui, A., "Propagation losses of silicon nitride waveguides in the near-infrared range," Appl. Phys. Lett. 86(12), 121111 (2005).

13. Yoon, D., D., Yoon, S., and Kim, Y., "Refractive index and etched structure of silicon nitride waveguides fabricate by PECVD," Thin Solid Films 12(515), 5004-5007 (2007).

14. Gorin, A., Jaouad, A., Grondin, E., Aimez, V., and Charette, P., "Fabrication of silicon nitride waveguides for visible-light using PECVD: a study of the effect of plasma frequency on optical properties," Opt. Express 16(18), 13509-13516 (2008).

15. Karouta, F., Vora, K., Tian, J., and Jagadishk, C., "Structural, composition and optical properties of PECVD silicon nitride layers," J. Phys. D: Appl. Phys. 45(44), 445301 (2012).

16. Goldbach, H. D., van der Werf, C. H. M., Löffler, J., Scarfo, A., Kylner, A. M. C., Stannowski, B., Arnoldbik, W. M., Weeber, A. W., Rieffe, H., Soppe, W. J., Rath, J. K., and Schropp, R. E. I., "Hot-wire chemical vapor deposition of silicon nitride for multicrystalline silicon solar cells," in [Conference Record of the Thirty-first IEEE Photovoltaic Specialists Conference], 1249-1252, IEEE (2005).

17. Mäckel, H. and Lüdemann, R., "Detailed study of the composition of hydrogenated SiNx layers for highquality silicon surface passivation," J. Appl. Phys. 92(5), 2602-2609 (2002).

18. Yin, Z. and Smith, F. W., "Optical dielectric function and infrared absorption of hydrogenated amorphous silcon nitride films: Experimental results and effective-medium-approximation analysis," Physical Review B 42(6), 3666-3672 (1990). 